\title{
eJRIEPS
}

Ejournal de la recherche sur l'intervention en éducation physique et sport

$25 \mid 2012$

Varia

\section{La gymnastique : un jeu de règles}

\section{Jean-François Robin}

URdition électronique

DOI : 10.4000/ejrieps.3750

ISSN : 2105-0821

\section{Éditeur}

ELLIADD

\section{Référence électronique}

Jean-François Robin, «La gymnastique : un jeu de règles », eJRIEPS [En ligne], 25 | 2012, mis en ligne le 01 janvier 2012, consulté le 10 décembre 2020. URL : http://journals.openedition.org/ejrieps/3750 ; DOI : https://doi.org/10.4000/ejrieps.3750

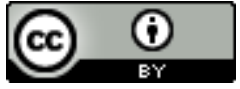

La revue eJRIEPS est mise à disposition selon les termes de la Creative Commons Attribution 4.0 International License. 
eJRIEPS 25 janvier 2012

\section{LA GYMNASTIQUE : UN JEU DE REGLES}

\section{Jean François Robin}

Institut National du Sport, de l'Expertise et de la Performance - Circeft/REV/EFA (EA n4384)

\section{Résumé}

Les règlements du jeu des activités gymniques et acrobatiques, appelés «codes de pointage ", sont modifiés régulièrement au profit d'une pratique toujours plus acrobatique. Une approche socio-anthropologique permet de les situer dans leur contexte. Les trois règles principales (difficulté, exécution, combinaison) sont décrites afin d'en comprendre les fondements et les transformations attendues. Elles auront des conséquences vis-à-vis de la motricité des gymnastes, des rôles sociaux de l'activité, enfin des émotions partagées. L'objectif de cet article est aussi d'identifier des conséquences des règlements pour l'intervention et de repérer des leviers qui permettent de «jouer » à la gymnastique de façon plus stratégique.

Mots clés : activités gymniques et acrobatiques, règlement du jeu, code de pointage, technologie.

\section{Introduction}

Dans les activités gymniques et acrobatiques, les règlements du jeu ont pour dénomination les «codes de pointage». Les codes de pointage regroupent les règles du jeu de la gymnastique. Son élaboration résulte d'un long processus inscrit dans notre histoire sociale. Quelles règles pour jouer à la gym ? Et par conséquent, à quelle gymnastique jouer aujourd'hui à l'école ? C'est à ces questions principales que cet article tente de répondre. Les codes de pointage sont considérés ici comme un outil à adapter en fonction des intérêts pédagogiques voulus par l'enseignant, en fonction des tensions didactiques spécifiques de l'activité (entre la prise de risque et sa maîtrise), enfin, en fonction des évolutions constatées dans les activités gymniques et acrobatiques. 


\section{eJRIEPS 25 janvier 2012}

Si le code de pointage permet d'apprécier un long «enchaînement » chez des experts, sa finalité est différente à l'école. La motricité des élèves gymnastes se décline essentiellement en termes d'actions gymniques et acrobatiques. Elles nécessitent une évaluation adaptée. La richesse de la motricité gymnique des élèves est en jeu et les règles du jeu existent pour la mettre en valeur.

\section{Caractéristiques sociologiques de la gymnastique}

Pour comprendre le règlement d'une activité sportive, il est utile de le replacer dans son contexte, c'est-à-dire dans celui de la pratique.

Issue d'une tradition militaire visant à former un soldat conquérant, la gymnastique fut d'abord utile aux démonstrations de force. Elle était statique, faite de postures et d'attitudes. Elle enseignait la force, le courage, la droiture ou l'abnégation. Après la deuxième guerre mondiale, la gymnastique devient plus aérienne ; les élans sont privilégiés pour construire une acrobatie encore peu maîtrisée du fait de conditions matérielles spartiates. Ce n'est que dans les années 80, qu'une gymnastique «plus aérienne, plus renversée et plus tournée » s'organise. Elle est depuis lors définitivement installée dans la famille des activités acrobatiques, qui comprend le trampoline, le tumbling, le plongeon, le ski acrobatique et bien d'autres. Paul Goirand montre que la gymnastique est passée d'une pratique d'élégance, imposée, collective et polyvalente à une pratique de risque, libre, individuelle et spécialisée (1996).

De nos jours, la gymnastique se pratique sous des formes variées (Goirand, 1994), du plus jeune âge (baby gym) à l'âge adulte avec une pointe en âge vers 9 ans. Le nombre de pratiquants ne cesse de décroître après la puberté (Robin, 1998). Nous remarquons que c'est à l'âge des élèves de collège que cette décroissance est la plus forte. Ces derniers ont peu de goût pour cette activité à l'école, quand elle est enseignée sous des formes traditionnelles. S’ils veulent construire des transformations durables chez leurs élèves, les enseignants pourraient s'orienter vers des formes novatrices de pratique.

Les formes de pratique sont également très diversifiées, d'une gymnastique de loisir, voire d'entretien, à une gymnastique de haute performance, d'une heure hebdomadaire à trentecinq heures d'entraînement par semaine. Ils sont ainsi plus d'un demi-million à s'adonner à 


\section{eJRIEPS 25 janvier 2012}

cette activité, en France, dans le cadre de fédérations sportives, scolaires ou affinitaires: UNSS, FFSU, FFGYM, FSCF, FSGT, UGSEL, UFOLEP, etc.

Aujourd'hui, la pratique de la gymnastique est, en France comme dans le monde, à plus de $80 \%$ celle des femmes ; ce qui nous fait dire que « la gymnastique est une activité physique féminine et pré pubère ".

L'intérêt d'une réflexion à propos des caractéristiques sociologiques de la gymnastique réside dans l'adéquation des propositions pédagogiques aux représentations sociales de l'activité. II s'agit également de mieux comprendre les transformations sociales et techniques de la pratique pour mieux s'adresser aux élèves. Enfin, le regard porté sur les populations de gymnastes permet d'adapter un règlement du jeu, qui provoquerait des transformations plus ajustées aux niveaux de pratique. Ce travail nécessite une analyse didactique préalable.

\section{L'intervention en sport}

Cette réflexion participe de celle développée en technologie des activités physiques et sportives, par Bouthier (2008) notamment. Elle vise à approcher les techniques corporelles, les étapes d'une démarche technologique et les situations de jeu comprises comme mise en scène et partage de l'expérience. Nous insistons, ici, sur les connaissances à propos des systèmes sociotechniques de production et de formation.

Cette réflexion à propos de l'usage des codes de pointage fait écho aux débats à propos de l'intervention. "Dans le domaine du sport, au sens large, l'intervention correspond à tout acte professionnel mettant en œuvre des compétences, des savoirs d'expérience et théoriques au service d'un objectif, dans différents champs (l'école, le club sportif, les loisirs actifs, la rééducation, la psychomotricité...), au profit de publics variés, aux différents âges de la vie " (Association pour la Recherche sur l'Intervention en Sport). II ne s'agit pas seulement de traiter de procédures d'évaluation, mais davantage de comprendre les conséquences explicites et implicites d'un règlement. Par ailleurs, l'activité de jugement est également une source de travail. Cet article a donc pour objectif de s'inscrire dans un débat scientifique ouvert aux controverses et de présenter quelques données issues de la recherche en didactique. L'idée consiste à éclairer le métier très complexe des intervenants du sport et de l'éducation physique avec quelques outils théoriques et pratiques pour mieux comprendre les 
eJRIEPS 25 janvier 2012

situations d'enseignement et d'apprentissage, pour mieux analyser la pratique des apprenants et pour proposer de nouvelles procédures d'intervention.

Nous nous intéressons à la première phase de la transposition didactique, où la question de la pratique sociale de référence se pose pour concevoir les pratiques, les savoirs et les correspondances scolaires. Nous croyons que les règlements du jeu constituent le premier savoir universel du sport dans la mesure où ils sont publics, formalisés, communiqués et qu'ils font l'objet d'un jugement social, qui s'inscrit dans des registres d'efficacité et d'utilité (Robin, 1998). A ce titre, le règlement peut servir de référence pour enseigner à des athlètes débutants comme à des plus experts.

\section{Les règles du jeu de la gymnastique : principes et originalités}

Fondamentalement dans les activités gymniques et acrobatiques, un règlement a pour fonction d'évaluer la performance, c'est-à-dire l'exploit, la prouesse, l'exceptionnel, l'extraordinaire. C'est en quelque sorte, ce qui ne peut jamais être atteint. Pendant longtemps en gymnastique, ce haut niveau de performance était symbolisé par la note 10, sans qu'on en sache vraiment l'origine. Depuis quelques années, sans doute sous le poids de l'évolution sociétale, le règlement a été modifié sans fixer de note plafond. Le règlement distingue les vainqueurs et vaincus et les hiérarchise dans leur construction de la performance. La fonction du règlement réside dans l'établissement de hiérarchies justes et indiscutables dès lors que les performances sont mesurées objectivement, que les règles sont respectées et que les arbitres sont neutres et indépendants.

Au-delà, un règlement est le garant des valeurs fondamentales du sport et de l'olympisme : "l'égalité des chances à l'inégalité du résultat », "la victoire symbolique toujours remise en jeu », « le respect réciproque des athlètes et des arbitres », etc. Le règlement définit dès lors le champion sportif comme symbole d'excellence sociale.

Comme leur nom l'indique, les codes de pointage ont pour but de "coder » le système d'attribution des "points ". Ils définissent les paramètres et les critères qui permettent de différencier les prestations des gymnastes. Ils servent à caractériser « les conditions de la victoire ». Parallèlement, les codes précisent aussi « les conditions du jugement »; c'està-dire, comment le collectif des juges doit s'organiser et ce que les juges sont habilités à évaluer. Il existe donc un certain nombre de données objectives et explicites, qui permettent 


\section{eJRIEPS 25 janvier 2012}

de poser une note. Celle-ci doit refléter avec rigueur et justesse le niveau de prestation des concurrents. Le regard porté sur les règlements publiés depuis 1936 (date du premier code de pointage international de la gymnastique) confirme une volonté permanente des législateurs de clarifier les critères d'appréciation, de les objectiver et de les distinguer.

\section{1. Première originalité}

Les règlements du jeu constituent les premiers savoirs de la discipline (cf. Robin, 1998. Enoncés propositionnels communiqués, publiés, décrits et objet d'un jugement social). En effet, dans le contexte des activités gymniques acrobatiques, les règlements du jeu font l'objet de publications internationales, qui servent de référence pour les juges et d'ouvrages pédagogiques pour les enseignants. Ceci constitue la première originalité des activités gymniques acrobatiques, où le règlement sert de référence pour l'acrobate, l'entraîneur, le juge et le spectateur. Cette volonté de mettre à la disposition des acteurs un outil de lecture de la gymnastique apparaît explicitement dans les intentions des rédacteurs des codes de pointage de la Fédération Internationale de Gymnastique. "La présente édition doit être largement adoptée comme aide-mémoire indispensable de tout juge, entraîneur, technicien et gymnaste " (Gander Arthur, Président de la FIG de 1966 à 1976, Code de pointage GAM, 1979). Tous les règlements sportifs n'ont pas cet objectif. II semble, cependant, utile d'identifier qui sont les utilisateurs pour penser les adaptations à venir. Pour jouer à la gymnastique, il est donc important de préciser dans un règlement « qui doit faire quoi ».

\section{2. Seconde originalité}

Paradoxalement, avec l'évolution du niveau technique, le règlement s'est progressivement coupé de la pratique du débutant. En effet, les codes de pointage internationaux sont devenus des outils d'évaluation pour la seule pratique du haut niveau. Aujourd'hui, il n'est plus possible pour un débutant de jouer à la gymnastique avec le même règlement du jeu que celui des champions. Par exemple, les éléments techniques hiérarchisés dans les grilles d'observation du code intègrent les techniques scolaires les plus difficiles (comme le salto ou le flip arrière) dans les catégories les moins valorisées. Des éléments fréquents à l'école (comme la roue ou la rondade) ne rapportent aucun point. La deuxième originalité du règlement du jeu met en évidence la nécessaire adaptation du règlement aux niveaux de pratique des athlètes. Pour jouer à la gymnastique, il est donc essentiel d'ajuster le règlement aux exigences de la pratique scolaire. 


\section{eJRIEPS 25 janvier 2012}

\section{Les règles du jeu essentielles de la gymnastique}

Les codes de pointage internationaux furent nombreux et souvent déclinés dans de nombreuses fédérations nationales (FSGT, FSCF, UFOLEP, USEP, UNSS, etc.) et internationales (FIST, FMI, etc.). Sur le principe, ils sont modifiés pour chaque nouvelle olympiade. Depuis les premiers travaux de Lapallu1, le règlement a gagné en objectivité. Les «codes» ont classé et déclassé, catégorisé, hiérarchisé des milliers de savoir-faire techniques. Ils ont affiné une définition de la « silhouette gymnique » acceptable par toutes et tous. Ils ont pris en compte les innovations techniques et matérielles, voire les ont provoquées. Enfin, ils sont l'enjeu de rivalités et de rapport de force internationaux. D'une façon générale, il s'agit donc d'un document qui évolue rapidement. Puisqu'il est contestable et modifiable, c'est un organe vivant.

Des permanences ont structuré le règlement, malgré cette transformation invariable. Des caractéristiques essentielles se dégagent, lesquelles alimentent une diversité de petites règles. Sur les traces de R. Deleplace (1983) et de P. Goirand (1990), les « poutres maîtresses » $d u$ règlement sont différenciées des règles secondaires. Ainsi pour la gymnastique, trois règles fondamentales articulent l'évaluation des prestations.

4. 1. La règle de la « difficulté »

* Le principe est le suivant : «plus le gymnaste sait faire de choses difficiles, plus il gagne de points ».

* Par exemple à l'Union Nationale du Sport Scolaire (fédération du sport scolaire français), l'élève gymnaste doit faire une rondade, un flip arrière, mais aussi un salto avant et un saut de mains pour assurer une note maximale en difficulté.

* Pour les pratiquant(e)s, la capacité à produire davantage d'acrobaties permet d'avoir plus de chances de victoire.

* II s'agit d'une logique additive.

* De fait, cette exigence propre aux activités acrobatiques, met en jeu leurs intégrités physique (ça peut faire mal) et psychique (ça peut faire peur). En effet, pour faire le meilleur

1 Lapallu Claude. Président de la direction technique permanente de la Fédération Française de Gymnastique (1948 à 1949), puis Président de la Fédération Internationale de Gymnastique (1949 à 1950). Premier rédacteur des règles du jeu de la gymnastique vers 1936. 


\section{eJRIEPS 25 janvier 2012}

score, les gymnastes doivent multiplier les prouesses aériennes. Ainsi, ils sont tenus de «prendre des risques ».

* Ce qui a fondamentalement évolué depuis un demi-siècle, c'est la construction de l'acrobatie, c'est-à-dire la multiplication des saltos et des vrilles. Elle devient toujours plus aérienne, plus renversée, plus manuelle, plus tournée.

* Si cette règle n'existait pas, alors la gymnastique ressemblerait davantage à une somme de déplacements simples et de formes élémentaires, sans doute laissant la place à des contenus chorégraphiques.

* C'est un bon scénario pour l'école de demain. Jouer à la gymnastique sous-entend « encourager la présentation d'acrobaties toujours plus complexes ».

4. 2. La règle de «l'exécution"

* Le principe est le suivant : "plus la production du gymnaste est maîtrisée, plus il gagne de points ».

* Pour les pratiquant(e)s, la capacité à réaliser avec facilité, grâce et aisance prouve leur maîtrise technique. La tenue des maintiens et des équilibres, l'amplitude des acrobaties, la qualité des formes de corps, la stabilité des réceptions sont des indicateurs pertinents pour évaluer cette aisance. C'est ce qui donne sens à l'esthétique gymnique.

* Les juges sont là pour apprécier le degré de maîtrise. Ils vérifient si, ce qui est présenté, est techniquement bien exécuté et maîtrisé. Chaque fois qu'une faute est identifiée, elle est pénalisée. II s'agit d'une logique soustractive. Le juge retire des points d'un total fixé par avance et qui a toute son importance.

* Ainsi, les gymnastes sont tenus de «contrôler les risques pris ". La technique favorise ce contrôle. II s'agit de faire techniquement juste ; ce qui a pour conséquence de "faire beau ", de « faire correct ». C'est le techniquement juste qui est beau.

* Cette règle a peu évolué depuis un demi-siècle. La recherche d'une motricité contrôlée est une permanence de l'activité.

* L'esprit de cette règle a pour but d'encourager la sécurité des pratiquant(e)s. Si elle n'existait pas, alors l'acrosport ressemblerait probablement à des jeux de cirque antique, où l'on verrait une surenchère acrobatique.

* C'est aussi un scénario pour l'école de demain. Jouer à la gymnastique sous entend « encourager la maîtrise d'acrobaties toujours plus complexes ». 


\section{eJRIEPS 25 janvier 2012}

4. 3. La règle des « exigences spécifiques»

* Le principe est le suivant: «plus le gymnaste se déplace dans un espace riche et varié, plus il gagne de points ». L'espace étant défini par l'appareil, le gymnaste et les rapports de l'un à l'autre, la règle exige des passages obligés, spécifiques à chaque agrès.

* Par exemple, le gymnaste doit passer par les deux faces de la barre et faire un élément sur la barre et un autre sous la barre. Le gymnaste est ainsi poussé à déployer une motricité la plus riche possible plus aérienne, plus manuelle, plus renversée, etc.

* Pour les pratiquant(e)s, la capacité à exploiter davantage le domaine de l'agrès permet d'avoir plus de chances de victoire.

* La logique est additive puisque plus le gymnaste répond aux exigences, plus il gagne de points.

* Cependant, cette règle met aussi en jeu leurs intégrités physique et psychique. Ils sont tenus de «prendre des risques» pour explorer toutes les faces et tous les niveaux de l'espace de l'agrès.

* Cette règle a beaucoup évolué depuis un demi-siècle. Elle conduit à coordonner davantage d'actions dans tous les plans de l'espace.

* Si cette règle n'existait pas, alors la gymnastique ressemblerait davantage à une somme d'aller-retour sur des engins aux dimensions réduites. C'était d'ailleurs le cas, lorsque la gymnastique a été pratiquée en salle et non plus en plein air, vers les années 30.

* Cette règle voit aussi son prolongement dans la pratique polyvalente ou spécialisée de la gymnastique. En effet, il n'est pas obligatoire pour un gymnaste de se présenter à tous les agrès, comme le renforce l'exemple des compétitions internationales, qu'elles soient par équipes ou individuelles. II existe une liberté de choix des engins. L'idée est proche du fonctionnement des sports collectifs où l'entraîneur fait entrer un joueur, qui développe particulièrement un champ d'expertise du jeu (ici, une exploitation très riche de l'espace de l'agrès). Cela encourage des stratégies nouvelles et pousse les gymnastes vers davantage de maîtrise technique.

* C'est un bon scénario pour l'école de demain. Jouer à la gymnastique sous-entend « encourager la variété d'acrobaties dans un espace toujours plus riche » sur des engins stratégiquement choisis par les élèves et l'enseignant. 


\section{eJRIEPS 25 janvier 2012}

\section{Les règles secondaires de la gymnastique}

5. 1. Les règles « explicites»

Les règles « secondaires » correspondent aux conventions qui permettent de départager les meilleurs (par exemple, les tenues obligatoires des filles et des garçons, ou encore, les registres de bonification). Parmi cet ensemble de données, quatre règles méritent une attention particulière, tant leurs conséquences pour les élèves sont sérieuses.

a) En premier lieu, il est utile de préciser «l'espace de jeu ». La gymnastique implique nécessairement la présence d'un appareil auquel le gymnaste est confronté. L'architecture de l'agrès a toute son importance.

Selon les époques les appareils sont différents et chaque appareil évolue. Par exemple, les dernières évolutions du cheval de saut concourent à un bond qualitatif des prestations. Dans la logique de l'activité, il fallait rendre cet agrès encore plus aérien, plus tourné, plus renversé et plus manuel. La table de saut est le fruit de ce défi. Elle apparaît pour la première fois en compétition, lors des Championnats du Monde de Gand (Belgique), en 2001. Elle est plus courte (1m20), plus large $(95 \mathrm{~cm})$, plus inclinée (79, plus rebondissante, mieux proté gée et mixte. De ce pont de vue, le matériel est une création didactique.

b) La « durée de la production » est une règle à définir si besoin est. La dialectique repose souvent sur la contradiction suivante : « plus il est exigé d'éléments techniques, moins ils sont difficiles »; ce qui va à l'encontre de la logique de l'activité.

c) Le «nombre de joueurs » est aussi un point utile à définir. A priori, la gymnastique sportive est considérée comme une activité individuelle, mais les gymnastes concourent principalement en équipe. Comme nous l'avons vu précédemment, il se développe des stratégies d'équipe. Cependant, il est concevable de pratiquer la gymnastique scolaire de façon plus collective (Amarouche et Nouillot, 1989). Les acrobaties synchronisées ou en cascade sont des formes possibles. Elles ont existé dans l'histoire de la gymnastique et encore aujourd'hui dans certaines fédérations affinitaires ou certaines modalités nouvelles de compétition (gymnaestrada, euroteam, etc.).

d) Pour la gymnastique au sol ou à la poutre, les « choix musicaux » peuvent avoir une utilité pour porter la chorégraphie, synchroniser les actions et faciliter des repères. En 


\section{eJRIEPS 25 janvier 2012}

aucun cas la musique est un fond sonore. Elle n'a rien d'obligatoire. C'est une contrainte supplémentaire, dont les termes peuvent être précisés pour s'inscrire dans un véritable projet culturel.

Modifier les termes des règles secondaires ne dénature pas fondamentalement l'activité. C'est davantage dans le but d'une transformation de la motricité que ces règles doivent être adaptées aux caractéristiques des gymnastes et aux finalités de la pratique.

\section{2. Les règles « implicites»}

Les activités gymniques et acrobatiques recèlent de règles implicites admises par tous. Par exemple, les droits d'action sur les joueurs ne sont pas légiférés contrairement à la majorité des activités sportives. Ainsi, rien n'interdit de frapper son concurrent, mais il ne viendrait à l'esprit de personne de le faire dans l'enceinte d'une compétition. Dans le même ordre d'idée, les gymnaestradas ne délivrent ni titre, ni classement, mais elles sélectionnent les meilleurs moments pour un gala de clôture où se retrouvent, en fait, les prestations les plus acrobatiques et les plus maîtrisées. II en va également ainsi des déplacements des gymnastes sur l'aire de compétition, de la présentation des juges et des équipes aux agrès, du silence et des encouragements des spectateurs ou encore du salut du gymnaste au corps de juges en début et en fin de prestation. Cela n'est écrit nulle part, mais fait partie du règlement admis tacitement et culturellement. Nous croyons nécessaire, en particulier chez les débutants, de mettre à jour cet implicite collectif pour faciliter la compréhension de l'esprit du jeu et la construction du règlement par les gymnastes.

II n'est pas rare de constater que des gymnastes, des professeurs d'EPS ou des entraîneurs méconnaissent les règles du jeu. Pour autant, les élèves jouent à une gymnastique peu acrobatique, qui reproduit les canons traditionnels d'une gymnastique statique et de postures.

\section{3. La dynamique des juges}

Dans les activités gymniques et acrobatiques, la performance est appréciée par des juges. L'évaluation est donc soumise à un jugement humain, dont les modalités sont précisées par le règlement. Une note devient alors le produit d'une négociation (David, 2003) entre les juges dans un souci d'intégrité, de justice, de justesse et d'équité. L'activité sociale des juges au travail fait dire à Durny (2011) que "la note finale ne se résume pas à une simple moyenne, mais résulte d'une dynamique issue de l'influence réciproque des juges et aussi du contexte de la compétition ». Un système de contraintes permet de garantir une volonté des 


\section{eJRIEPS 25 janvier 2012}

juges de respecter l'égalité entre les concurrents à défaut d'une utopique neutralité. La dimension humaine du jugement prête parfois le flan à des dérives (tricherie, coalition, pression), mais révèle aussi toute la difficulté du travail des juges. Pour jouer à la gymnastique, il est donc nécessaire de permettre aux gymnastes de passer par les différents rôles sociaux de l'activité (gymnaste, juge, entraîneur, spectateur) pour en comprendre les mécanismes et les errances.

\section{La contradiction née du règlement}

Les trois règles essentielles, qui viennent d'être décrites, ont fondamentalement des conséquences sur la motricité. C'est parce qu'il y a ces règles-là, qu'il existe une motricité gymnique aussi acrobatique.

Mais du règlement découle aussi toute la logique de l'activité. Ainsi, les deux règles, dites de "difficulté » et "d'exigences spécifiques ", poussent les gymnastes à prendre des risques. Ils sont tenus de tenter davantage de prouesses. Contradictoirement, la règle de «l'exécution » contribue à la maîtrise des risques. Les gymnastes sont tenus de contrôler ce qu'ils tentent.

Le règlement est donc adroit (“ ad-directus ", c'est à dire " dans la bonne direction ", là où on veut mener le gymnaste) puisqu'il pénalise des enchaînements qui seraient soit trop dangereusement acrobatiques, soit trop peu acrobatiques. L'anti-jeu serait de limiter les prestations à des contenus ni renversés, ni manuels, ni aériens ou de présenter des prouesses ponctuées de chutes et de blessures. Ce type d'activités humaines existe par ailleurs, mais ne relève pas de la gymnastique.

La gymnastique propose donc de "gérer" cette contradiction entre la prise de risque inhérente à l'activité acrobatique et la maîtrise du risque nécessaire à la sécurité du pratiquant. Cette "unité contradictoire " (Goirand, 1987) caractérise la logique de l'activité acrobatique en même temps qu'elle en révèle les limites.

Selon le rôle social occupé pour faire vivre la gymnastique, la gestion de cette contradiction essentielle ne s'administre pas de la même façon.

* Dans la gymnastique fédérale de haute performance, le gymnaste n'a pas d'autres choix que d'apprendre toujours davantage d'acrobaties et de tenter de les maîtriser lors des 


\section{eJRIEPS 25 janvier 2012}

confrontations avec ses partenaires et contre ses adversaires. II est particulièrement dans une logique de prise de risque.

* Le juge a pour seule mission de vérifier la maîtrise des réalisations, le jour " $\mathrm{J}$ ", à un instant « $t$ ». Son point de vue à propos du degré d'habileté est tranché. II constate l'écart entre les exigences du code de pointage et les prestations, ceci dans une logique de maîtrise des techniques gymniques.

* Le spectateur apprécie ce qui est " extraordinaire et harmonieux ", c'est-à-dire ce qui est féerique (Jeu, 1977). S'il est le plus souvent incompétent pour juger de la technique, il différencie celles et ceux qui restent longtemps en l'air et qui tournent vite. II s'attache aussi à celles et ceux qui renvoient une impression d'aisance et de contrôle. II ne vient pas pour constater une hécatombe de chutes... II est surtout dans une émotion née de la logique de maîtrise et d'élégance.

* L'entraîneur est traditionnellement celle ou celui qui décide de la meilleure option : " faire difficile » ou « faire maîtrisé ». Contrairement à la majorité des activités sportives, ici, ce n'est pas le joueur qui décide dans l'action, mais l'entraîneur qui tranche en amont de la prestation. Nous constatons que les choix sont souvent réalisés en dépit de l'adversité, qui reste souvent méconnue des entraîneurs même des plus performants (Robin, 2011).

La gymnastique n'est donc pas à réduire à une activité traditionnelle de maîtrise de formes de corps techniques. Elle est une activité humaine dynamique, qui introduit des stratégies variées entre différents protagonistes. Cette vitalité existe si la contradiction «prise de risque / maîtrise du risque » dans le règlement laisse possible des choix variés.

\section{Les stratégies des gymnastes et de leurs entraîneurs}

Mais pour gagner, faut-il faire « plus difficile » ou faut-il faire « plus maîtrisé »?

En fait, selon la distribution des points dans les trois secteurs du règlement de l'activité, les stratégies peuvent être adaptées. En gymnastique, le règlement est relativement équilibré puisque selon le code de pointage, les valeurs se répartissent, aujourd'hui, globalement autour de $50 \%$ pour la difficulté et les exigences spécifiques et $50 \%$ pour la maîtrise. Ainsi, pour gagner au jeu de la gymnastique, trois solutions sont concevables :

a) Le joueur fait plus "difficile » que les autres (au sens ou il fait plus d'acrobaties ou qu'il en fait de plus complexes). 


\section{eJRIEPS 25 janvier 2012}

b) Le joueur fait plus «beau » que les autres (au sens ou il maîtrise les réceptions, les maintiens, les attitudes et les postures). De ce point de vue, il développe une technique mieux adaptée aux exigences de l'activité.

c) Le joueur fait plus « difficile » et plus « beau » que les autres.

Cette conception des stratégies du jeu gymnique se rapproche de l'idée de «rovisation de l'activité » telle que Paul Goirand l'avait défini (1990). Les notions "d'originalité » et de "virtuosité » restant pour nous peu opérationnelles, nous préférons conserver cette terminologie.

\section{Quelles stratégies du jeu?}

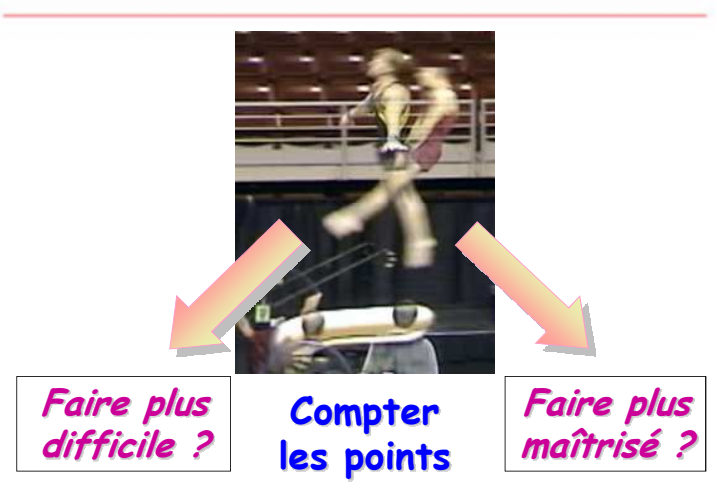

Le constat est fait de stratégies du jeu induites par le règlement lui-même. La répartition des points ne se fait donc pas au hasard; elle produit des effets attendus sur la motricité des gymnastes.

\section{Les règles du jeu comme variables didactiques}

Une variable didactique, c'est la condition « qui dans les situations d'apprentissage provoque, lorsqu'on agit sur elle, des adaptations, des régulations, des changements de stratégies, et qui finalement, permet de faire avancer la notion en construction" (Astolfi, 1990, p. 10). Modifier certaines règles du jeu, tant du point de vue du principe de la règle que de sa valeur, transforme la motricité des pratiquants. Ainsi, la modification de la règle agit comme une variable didactique. En agissant sur un paramètre, nous provoquons des progrès chez les 


\section{eJRIEPS 25 janvier 2012}

apprenants. II est donc nécessaire d'identifier quelle règle agit sur quelle dimension de la motricité.

a) Que provoque une «sur évaluation » de la règle de la « difficulté »? A priori, donner plus de points à ce secteur de la note incite les gymnastes à présenter davantage d'éléments techniques difficiles. Cette somme de savoir-faire permet d'évaluer des progrès quantitatifs. Elle nécessite l'élaboration d'un « référentiel commun », c'est à dire d'un inventaire des techniques repérées, de leur terminologie et de leur valeur. Cette " sur évaluation » peut être utile lorsque l'enseignant ou l'entraîneur souhaite valoriser la prise de risque de ses élèves.

b) Que provoque une «sur évaluation » de la règle de «l'exécution »? A priori, donner plus de points à ce secteur de la note encourage les élèves à maîtriser davantage les éléments techniques. Cette évaluation cible les progrès qualitatifs. Elle nécessite de connaître les pénalités et leur valeur. Cette «sur évaluation » peut être utile lorsque l'enseignant ou l'entraîneur souhaite valoriser les exigences de tenue du corps. Les points peuvent représenter jusqu'à $75 \%$ de la note.

c) Que provoque une «sur évaluation » de la règle des « exigences spécifiques »? Plus l'espace investigué est riche, mieux les gymnastes sont notés. Cette évaluation cible un progrès qualitatif. Elle nécessite de définir les exigences et leur valeur.

Cette «sur évaluation » peut être utile lorsque l'enseignant ou l'entraîneur souhaite valoriser les déplacements dans tout l'espace de l'engin. Les points peuvent représenter jusqu'à $25 \%$ de la note.

Comprendre la règle du jeu comme une variable didactique, c'est une démarche conforme à celle adoptée plus généralement en EPS (Boda et Récopé, 1991).

\section{Conclusion}

Les règles de la gymnastique ont pour objectifs de permettre aux gymnastes de mieux jouer ensemble. Comme dans tout jeu sportif, elles servent aussi à hiérarchiser les joueurs, peutêtre plus que dans d'autres activités. Elles visent enfin à respecter les intégrités physique et psychique des joueurs. II semblerait donc inapproprié d'ignorer le règlement lorsque cette activité doit être enseignée à l'école.

Pour les professeurs d'EPS, l'analyse des codes de pointage permet d'en comprendre les fondements, les principes, les valeurs et les usages. Elle facilite surtout le choix de stratégies 


\section{eJRIEPS 25 janvier 2012}

adaptées aux enjeux de formation. Ainsi, si les élèves doivent devenir des gymnastes « stratèges », alors ils doivent avoir accès à la compréhension du règlement et participer à leur élaboration.

A des fins d'enseignement, la règle du jeu peut être conçue comme une variable didactique. Elle désigne alors les possibilités de variation qui sont échafaudées par un enseignant dans l'organisation des conditions d'apprentissage. Le but de cet article consiste à donner chair à cette approche « pédagogique et didactique » du règlement.

Les activités gymniques et acrobatiques offrent aux élèves des savoir-faire techniques originaux. «Apprendre à piloter son corps dans l'espace » nécessite pour les gymnastes de savoir pour quoi le faire.

Nous croyons que l'étude des codes de pointage, compris comme les fondements des activités gymniques et acrobatiques, est nécessaire pour mieux former les pratiquants, du débutant à l'expert. Cela nécessite une analyse technologique à des fins didactiques. Une épistémologie des techniques est une option pertinente pour étudier scientifiquement l'origine logique des techniques, leur valeur, leur portée et leur sens. Les codes de pointage pourraient alors constituer des objets scientifiques.

\section{Bibliographie}

Amarouche, P. et Nouillot, P. (1989). Objectif gym : vivre à l'heure des interclasses. Dans L'éducation physique et sportive, aujourd'hui ce qui s'enseigne (p. 101-104). Editions du SNEP.

Astolfi, J. P. (1990). L'important, c'est l'obstacle. Cahiers pédagogiques, 281.

Boda, B. et Récopé, M. (1991). Instrument d'analyse et de traitement de l'APS à des fins d'enseignement. Revue EP.S., 231, 56-58.

Bouthier, D. (2008). L'approche technologique et la didactique professionnelle. Communication présentée lors de la 1ère rencontre suisse sur l'intervention en éducation physique et sport : «Apport des courants de la recherche actuels pour la formation ». HEP Vaud Lausanne.

David, B. (2003). Évaluation et notation en éducation physique et sportive. Regard sur la formation et perspectives de recherche. Paris: INRP. Collection : documents, travaux, recherche. ISBN : 2-7342-0945-4. 


\section{eJRIEPS 25 janvier 2012}

Deleplace, R. (1983). La recherche sur la spécialisation, l'entraînement, la performance. Dans La recherche en STAPS (p. 93-151). Nice 19-20 Sept. 1983.

Durny, A. (2011). Comment envisager la conception de programmes de formation des arbitres et juges à partir d'une analyse de leur activité effective. Dans F. Dosseville et S. Laborde (Eds.), Les différentes facettes de l'arbitrage. Publibook.

Goirand, P. (1987). Une problématique complexe : des pratiques sociales des APS aux contenus d'enseignement en EPS. SPIRALES, 1, complément, 11-14.

Goirand, P. (1990). Didactique de la gymnastique et EPS. Dans EP et didactique des APS. Édition de l'AEEPS (p. 74-87).

Goirand, P. (1994). Des pratiques sociales en gymnastique aux pratiques scolaires. SPIRALES, 7, 155-157.

Goirand, P. (1996). Evolution historique des objets techniques en gymnastique. Dans P. Goirand et J. Metzler (Dir.), Techniques sportives et culture scolaire (p. 99-144). Paris :Éditions revue EPS.

Jeu, B. (1977). Sport, émotion, espace. Paris : Vigot.

Robin, J. F. (1998). Transposition didactique en gymnastique: les savoirs de référence développés par quatre leaders de théories didactiques. Dans C. Amade-Escot et col (Eds.), Recherches en EPS: bilan et perspectives (p. 109-118). Paris: Editions Revue EPS.

Robin, J. F. (à paraître). Analyse des gestes professionnels des entraîneurs de l'équipe de France féminine de gymnastique lors de l'Olympiade 2004-2008. Actes du colloque FKQ / ARIS de mai 2010. 